\title{
DNA methylation and retinal degenerative diseases: at the crossroad between genes and diet*
}

\author{
Andrea Maugeri ${ }^{1 \dagger}$ \\ ${ }^{1}$ Department of Medical and Surgical Sciences and Advanced Technologies \\ 'G.F. Ingrassia', University of Catania, Italy
}

\begin{abstract}
Summary
Retinal degenerative diseases are the leading causes of blindness and low vision among working-age and older adults worldwide, with 170 and 130 million individuals suffering from age-related macular degeneration (AMD) and diabetic retinopathy, respectively. Although several studies began to show benefits from dietary interventions against retinal degenerative disease, an integrated approach is needed to understand molecular mechanisms underpinning the protective or risky effect of dietary factors. A specific area of research that elucidates mechanisms involved in gene-diet interaction is the Nutri-epigenomics, the study of the impact of diet on gene expression by modulating epigenetic mechanisms. The present research investigated the role of DNA methylation - one of the most commonly analysed epigenetic mechanisms - in the pathophysiology of retinal degenerative diseases, by exploiting a multiple integrated approach. In vitro studies initially helped us to understand how pathological features of retinal degeneration (e.g. oxidative stress, inflammation and hyperglycaemia) modulated functions of enzymes involved in the methylation of Long Interspersed Nuclear Element 1 (LINE1) sequences in retinal cells. We also proved that some nutrients (e.g. resveratrol and curcumin) might counteract these effects and restore DNA methylation level in retinal cells under oxidative, inflammatory and high glucose conditions. We further analysed whether LINE-1 methylation level differed between patients with AMD and controls without posterior segment eye diseases. Interestingly, we noted a significant difference between the two groups, with higher LINE-1 methylation level in blood samples from AMD patients. This evidence — albeit promising for biomarker discovery- requires
\end{abstract}

*Abstract of one of the Best PhD Theses Award, 2020.

$\dagger$ E-mail: andrea.maugeri@unict.it 
confirmation by further large-size prospective studies taking into account different factors. Our research, in fact, also suggested that the risk of retinal degenerative diseases derives from the combination of genetic risk variants, clinical characteristics, environmental exposures and unhealthy lifestyles, which in turn are interrelated. Thus, it would be interesting to study how the exposome - the totality of exposures individuals experience over the course of life- might induce epigenetic mechanisms able to reduce or increase the risk for retinal degenerative diseases.

Keywords: Retinal disease, epigenetic mechanisms, diet.

\section{Riassunto \\ Metilazione del DNA e malattie degenerative della retina: un incrocio di geni e dieta*}

Le malattie degenerative della retina sono tra le principali cause di cecità e visione ridotta tra gli adulti in età lavorativa e in tarda età, con 170 e 130 milioni di individui che soffrono di degenerazione maculare dovuta all'età (age-related macular degeneration, AMD) e retinopatia diabetica, rispettivamente. Sebbene diversi studi comincino a mostrare vantaggi di interventi dietetici sulla malattia degenerativa retinica, occorre un approccio integrato per comprendere i meccanismi alla base degli effetti protettivi o di rischio dei fattori dietetici. Un ambito specifico della ricerca volta a spiegare i meccanismi coinvolti nell'interazione fra geni e dieta è la nutri-epigenomica, ossia lo studio dell'impatto della dieta nell'espressione genetica attraverso meccanismi epigenetici. Il presente lavoro di ricerca ha investigato il ruolo della metilazione del DNA — uno tra i meccanismi epigenetici studiati più comunemente - nella patofisiologia delle malattie degenerative retiniche, mediante un approccio multiplo integrato. Studi in vitro hanno inizialmente consentito di comprendere come le caratteristiche patologiche della degenerazione retinica (ad esempio, lo stress ossidativo, l'infiammazione e l'iperglicemia) modulino funzioni di enzimi coinvolti nella metilazione di sequenze LINE-1 (Long Interspersed Nuclear Element 1) nelle cellule retiniche. Abbiamo altresì dimostrato che alcune sostanze nutritive (ad esempio il resveratrol e la curcumina) possano contrastare tali effetti e ripristinare il livello di metilazione del DNA in cellule retiniche in condizioni di ossidazione, infiammazione, e di elevata concentrazione di glucosio. Abbiamo inoltre analizzato l'eventuale differenza di livello di metilazione delle LINE-1 in pazienti con AMD e controlli in assenza di malattie del segmento oculare posteriore. È interessante notare una differenza significativa fra i due gruppi, con elevato livello di metilazione delle LINE-1 in campioni ematici da pazienti AMD. Tale evidenza - per quanto promettente per la scoperta di biomarcatori- richiede conferma da parte di studi su più larga scala, che tengano in conto diversi fattori. La nostra ricerca, infatti, suggerisce altresì che il rischio di malattie retiniche degenerative derivi dalla combinazione di varianti di rischio genetico, specifiche cliniche, esposizione a parametri ambientali, e stili di vita malsani, tra loro correlati. Pertanto, sarà interessante studiare come l'esposoma

*Riassunto di una delle Migliori Tesi di Dottorato premiate nel 2020. 
— la totalità di esposizioni che un individuo sperimenta durante la propria vita — possa indurre meccanismi epigenetici atti a ridurre o incrementare il rischio di malattie retiniche degenerative.

Parole chiave: Malattie della retina, cause epigenetiche, dieta. 